\title{
A MODULATION SOLUTION OF THE SIGNALLING PROBLEM FOR THE EQUATION OF SELF-INDUCED TRANSPARENCY IN THE SINE-GORDON LIMIT
}

\author{
A. A. Minzoni and N. F. Smyth
}

\begin{abstract}
In the present work, modulation theory is used to study the signalling problem for the equations of self-induced transparency in the Sine-Gordon limit. In the case in which the signal switches on instantaneously to a finite value, the modulation solution is found to be a modulated kink train. To construct this train the modulation theory of Forest and McLaughlin for the Sine-Gordon equation is used, and the solution is found to be the analogue of the Gurevich-Pitaevskii solution for the Korteweg-de Vries equation. The modulation theory solution is compared with a full numerical solution of the Sine-Gordon equation, and good agreement is found.
\end{abstract}

\section{Introduction}

The phenomenon of self-induced transparency arises when coherent light interacts resonantly with the transition frequency of two populated energy levels of the material through which the light is propagating. In this situation, the light can propagate as a soliton pulse and depending on the intensity and duration of the incident pulse, many solitons can form. Details of this phenomenon can be found in Lamb [5]. The model used for the propagation of the light through the material treats the electromagnetic wave in a classical manner, and the interaction of the light with the energy levels of the material is treated using quantum mechanics on approximating the atoms as two level atoms (in other words, the other energy levels play no role in the interaction process). The incident wave is described as a slowly varying monochromatic wave with envelope amplitude $E$ and phase $\phi$. The interaction of the light with the material is via the dipole moment of the atoms and the incident field. The use of quantum mechanics then provides an expression for the polarisation of the material in terms of the envelope amplitude and phase. In this manner, equations for the modulation of the electric field of the light coupled with Schrödinger's equation for the two level atom are obtained. These equations can be written in terms of the envelope amplitude, one component of the polarisation and the population density difference between the two energy levels. If the atoms are assumed to be stationary, then it can be shown that the envelope amplitude is related to the time derivative of the solution of the Sine-Gordon equation.

The signalling problem for the Sine-Gordon equation can be solved using the inverse scattering method when the incident signal has a finite duration time [5]. As the duration time of the signal increases, more and more solitons are generated, which

Received March 29, 1996, revised June 25, 1996.

1991 Mathematics Subject Classification: 35L50,35Q60,78A99.

Key words and phrases: modulation, Sine Gordon equation. 
suggests that a modulated periodic wavetrain solution is a good approximation to the exact solution when the duration time of the input signal is large (or infinite).

To study this possibility, the modulation theory of Whitham [8] is used. Modulation theory for the Sine-Gordon equation has been developed by Forest and McLaughlin [2] from the inverse scattering solution for the Sine-Gordon equation. Using the theory of Riemann surfaces, they derived the Riemann invariant formulation of the modulation equations for multi-phase modulated wavetrains. In the present work, the special case of a one-phase wavetrain is considered, and an explicit centered simple wave solution of these modulation equations is found. It is shown that this simple wave solution can be adjusted to produce a modulated wave solution which satisfies the required boundary conditions for the self-induced transparency problem. This modulation theory complements the result from inverse scattering for the case of a signal of finite duration [4]

\section{Formulation and modulation equations}

The equations for self-induced transparency are considered for the electric field $E$, the inverted population $N$, and the polarisation $S$ as given by Lamb [5] in the form of a signalling problem

$$
\begin{gathered}
\frac{\partial E}{\partial t}+c \frac{\partial E}{\partial x}=\Omega^{2} S, \\
\frac{\partial S}{\partial t}=E N, \quad \frac{\partial N}{\partial t}=-E S,
\end{gathered}
$$

for $x>0$ and $t>0$, together with the initial conditions

$$
E(x, 0)=0, \quad S(x, 0)=0, \quad N(x, 0)=1, \quad x \geq 0,
$$

and the signalling condition

$$
E(0, t)=E_{0} \text {. }
$$

The system (1) forms a hyperbolic system with a right-going characteristic and vertical characteristics. The signalling problem hence is given on the right-going characteristic.

Due to the form of the characteristics, it is convenient to introduce the signalling coordinates

$$
\xi=\frac{\Omega}{c} x, \quad \tau=\Omega\left(t-\frac{x}{c}\right)
$$

and the change of dependent variables

$$
S=-\sin \phi, \quad N=-\cos \phi, \quad E=\frac{\partial \phi}{\partial t} .
$$

With these new variables, the system (1) to (3) is transformed into the Sine-Gordon equation

$$
\frac{\partial^{2} \phi}{\partial \tau \partial \xi}=-\sin \phi, \quad \xi>0, \quad-\infty<\tau<\infty
$$

together with the boundary conditions

$$
\frac{\partial \phi}{\partial \tau}= \begin{cases}E_{0} & \text { on } \xi=0, \tau \geq 0 \\ 0 & \text { on } \xi=0, \tau<0\end{cases}
$$


Since the modulation theory of Forest and McLaughlin [2] has been developed in laboratory coordinates, the new change of variables

$$
u=\tau+\xi, \quad v=\tau-\xi
$$

is introduced to obtain the equation

$$
\frac{\partial^{2} \phi}{\partial v^{2}}-\frac{\partial^{2} \phi}{\partial u^{2}}+\sin \phi=0
$$

with the boundary conditions

$$
\frac{\partial \phi}{\partial u}+\frac{\partial \phi}{\partial v}= \begin{cases}E_{0} & \text { for } u=v, v \geq 0 \\ 0 & \text { for } u=v, v<0\end{cases}
$$

This system of equations can be interpreted as a signalling problem for an equation for a nonlinear string [5]. The disturbance moves along the right-going characteristic $u=v$ and sheds waves moving on the left-going characteristics $u+v=$ const.

In order to apply modulation theory to the boundary-value problem of concern in the present work, we need to recall briefly the results of Forest and McLaughlin [2]. The relevant results from that work are the ones for the kink trains described on page 44 of [2]. In [2], it was shown how multiply periodic solutions of the Sine-Gordon equation can be parametrised in terms of a Riemann surface associated with the periodic Lax Pair for the Sine-Gordon equation. In that representation, the parameters of the family of multiply periodic solutions are branch points on the (two sheeted) surface. It then was shown that the infinite sequence of conservation equations for the Sine-Gordon equation could be obtained as the coefficients in the expansion of a certain analytic function on the Riemann surface when the variable (on the surface) goes to infinity. In those terms, modulation theory was formulated by allowing the branch points to depend on slow space and time variables and then averaging out the fast phase variable. Conservation equations for the slow dependent variables (the parameters of the multi-phase wavetrain) then were found to result. It is remarkable that the form of the equations is explicit. Since the conservation equations were obtained from an analytic function on the Riemann surface, it follows from the general theory of such functions that in order to satisfy the conservation equations on the whole surface, it is sufficient to satisfy them at the branch points. This last requirement gave the Riemann invariant form of the modulation equations. This formulation of modulation theory for the Sine-Gordon equation now will be used to discuss the boundary-value problem described above. To this end, two negative real numbers $E_{1}<E_{2}<0$ are introduced. They define the branch points of the surface. We also consider the canonical cycle $b$ on the surface whose projection is shown in Figure 1. form

In these variables, the single phase travelling wave solution is represented in the

$$
e^{-i \Psi(u, v)}=\frac{\mu(u, v)}{\sqrt{E_{1} E_{2}}}
$$

where $|\mu|=\sqrt{E_{1} E_{2}}$ and where $\mu$ satisfies the differential equation

$$
\frac{d \mu}{d \theta}=-2 i C \sqrt{\mu\left(\mu-E_{1}\right)\left(\mu-E_{2}\right)} .
$$




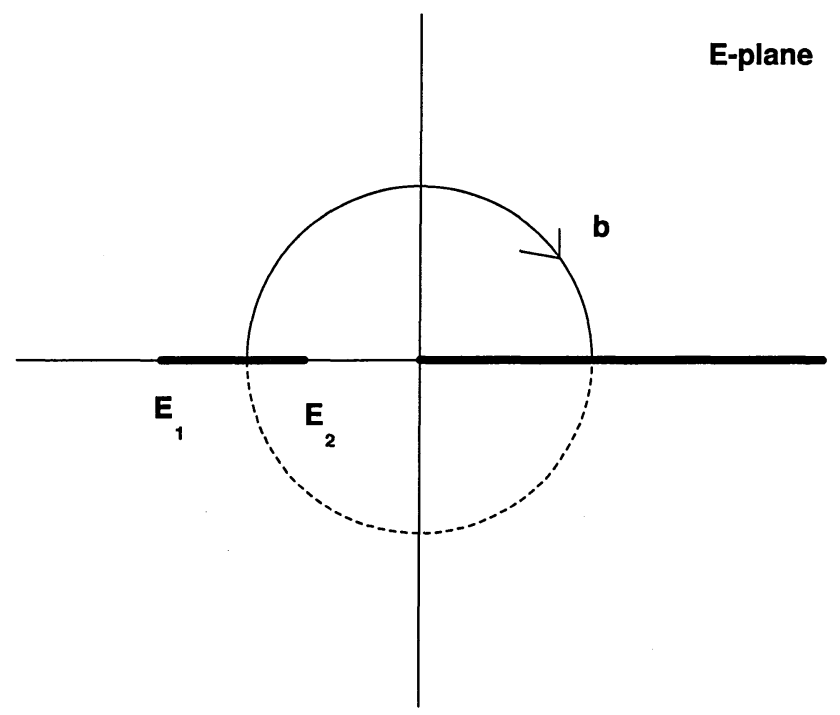

Figure 1. Complex $E$-plane

The branch cuts for the square root in this equation are shown in Figure 1. The normalisation constant $C$ is defined by

$$
C=-\frac{1}{4 \pi i} \int_{b} \frac{d \mu}{\sqrt{\mu\left(\mu-E_{1}\right)\left(\mu-E_{2}\right)}} .
$$

With this normalisation, the function $\exp (-i \Psi)$ is $2 \pi$-periodic with phase

$$
\theta=\frac{1}{C}(\kappa u+\omega v)
$$

where the wavenumber $\kappa$ and frequency $\omega$ are given by

$$
\kappa=\left(1-\frac{1}{16 \sqrt{E_{1} E_{2}}}\right) \text { and } \omega=\left(1+\frac{1}{16 \sqrt{E_{1} E_{2}}}\right) .
$$

The wavetrain is modulated by allowing the two parameters $E_{1}$ and $E_{2}$ to be slowly varying functions. Using the representation described above, Forest and McLaughlin [2] found that the modulation equations for a single phase wavetrain for the SineGordon equation take the simple Riemann invariant form

$$
\begin{aligned}
& \frac{\partial E_{1}}{\partial v}-S^{(1)} \frac{\partial E_{1}}{\partial u}=0 \\
& \frac{\partial E_{2}}{\partial v}-S^{(2)} \frac{\partial E_{2}}{\partial u}=0
\end{aligned}
$$

where the characteristic speeds $S^{(1)}$ and $S^{(2)}$ are real and are given by

$$
S^{(l)}=\frac{E_{l}+\frac{1}{16} \sqrt{E_{1} E_{2}} E_{l}^{-1}-\left(C^{+}+\frac{1}{16} \sqrt{E_{1} E_{2}} C^{-}\right)}{E_{l}-\frac{1}{16} \sqrt{E_{1} E_{2}} E_{l}^{-1}-\left(C^{+}-\frac{1}{16} \sqrt{E_{1} E_{2}} C^{-}\right)}
$$


and the functions $C^{+}$and $C^{-}$are determined by the condition that integrals of the differentials

$$
\begin{aligned}
& \Omega_{+}=-\frac{1}{2}\left(E-C^{+}\right) \frac{d E}{\sqrt{E\left(E-E_{1}\right)\left(E-E_{2}\right)}} \\
& \Omega_{-}=-\frac{\sqrt{E_{1} E_{2}}}{2}\left(E^{-1}-C^{-}\right) \frac{d E}{\sqrt{E\left(E-E_{1}\right)\left(E-E_{2}\right)}}
\end{aligned}
$$

along the $b$-cycle vanish.

In order to use the modulation solution outlined above to solve (9) and (10), we recall that the travelling wave solution $\Psi(u, v)$ has phase velocity greater than one. However, it is easily seen that $\phi(u, v)=\pi+\Psi(v, u)$ solves the same equation, but now the wavetrain has a phase velocity less than one. Furthermore, the modulation equations for $\Psi(v, u)$ are just (16) with $u$ and $v$ interchanged. Therefore $E_{1}$ and $E_{2}$ now satisfy

$$
\begin{aligned}
& \frac{\partial E_{1}}{\partial u}-S^{(1)} \frac{\partial E_{1}}{\partial v}=0, \\
& \frac{\partial E_{2}}{\partial u}-S^{(2)} \frac{\partial E_{2}}{\partial v}=0,
\end{aligned}
$$

for the transformed variables. With these transformed equations the qualitative behavior of the modulation solution now can be described. To do this it is convenient to parameterise $\mu$ in terms of the real variable $y(\theta)$ in the form

$$
\mu=\sqrt{E_{1} E_{2}} e^{-2 i \arctan y}, \quad \phi=2 \arctan y .
$$

Since the constant $C$ in (13) is real and positive, we take $y$ to be of the form $y=y(x), x=C \theta$. Substituting this form into (12) for $\mu$ and using (20) gives the equation for $y$

$$
\left(\frac{d y}{d x}\right)^{2}=2 \sqrt{E_{1} E_{2}}\left(1-y^{4}\right)-\left(E_{1}+E_{2}\right)\left(1+y^{2}\right)^{2} .
$$

It is clear from this equation that as $E_{2} \rightarrow E_{1}$, the solution for $\phi$ is a soliton since in this limit, the quartic on the right-hand side of (21) has co-incident roots. On the other hand, as $E_{2} \rightarrow 0$, we have

$$
\frac{d y}{d x}=\sqrt{-E_{1}}\left(1+y^{2}\right)
$$

It then follows that in this limit

$$
\phi \sim \pi+2 \sqrt{-E_{1}}\left[\left(1-\frac{1}{16 \sqrt{E_{1} E_{2}}}\right) v+\left(1+\frac{1}{16 \sqrt{E_{1} E_{2}}}\right) u\right],
$$

and the behavior of the solution is singular. However, the physical variable is the electric field which is given by $\phi_{u}+\phi_{v}$ (see (4), (5), and (8)), not $\phi$, and this combination is regular. To leading order, the electric field is then

$$
E=\frac{\partial \phi}{\partial u}+\frac{\partial \phi}{\partial v} \sim 4 \sqrt{-E_{1}}
$$

From the behavior of the single phase wavetrain solution deduced above, it is clear that the relevant centered simple wave solution of the modulation equations needed for our boundary-value problem is the one with the Riemann invariant $E_{1}$ constant and 
with the expansion fan occurring on the Riemann invariant $E_{2}$. The Riemann invariant $E_{2}$ changes from $E_{2}=0$ on the leading characteristic $u=v$ to the value $E_{2}=E_{1}$ on the trailing characteristic of the expansion fan. To show that this construction is possible, we need to show that $S^{(2)} \rightarrow 1$ as $E_{2} \rightarrow 0$ and that $S^{(2)}$ coincides with the reciprocal speed of the left-going leading soliton of the expansion fan as $E_{2}$ decreases to $E_{1}$ (note that solitons propagate to the left in the phase variable (14)).

We therefore consider the simple wave solution $E_{1}=$ constant and $E_{2}=f(u / v)$ of the modulation equations (19). From (19), it can be seen that the simple wave solution then is implicitly defined by

$$
\frac{u}{v}=-\frac{1}{S^{(2)}\left(E_{2}\right)}
$$

It only remains to verify that the function $S^{(2)}$ has the correct behavior and is monotone.

To study the function $S^{(2)}$, the conditions (18) determining $C^{+}$and $C^{-}$are written in the form

$$
C^{+}=\frac{\int_{b} \frac{E d E}{\sqrt{E\left(E-E_{1}\right)\left(E-E_{2}\right)}}}{\int_{b} \frac{d E}{\sqrt{E\left(E-E_{1}\right)\left(E-E_{2}\right)}}}, \quad C^{-}=\frac{\int_{b} \frac{d E}{E \sqrt{E\left(E-E_{1}\right)\left(E-E_{2}\right)}}}{\int_{b} \frac{d E}{\sqrt{E\left(E-E_{1}\right)\left(E-E_{2}\right)}}} .
$$

Then, as $E_{2} \rightarrow 0$, the integrals defining $C^{+}$and $C^{-}$are finite since the pole at $E=0$ is inside the contour $b$ (see Figure 1). We thus have from (17) that, as $E_{2} \rightarrow 0$,

$$
-\frac{1}{S^{(2)}} \sim-\frac{\frac{1}{16} \sqrt{\left|E_{1}\right|} \frac{1}{\sqrt{\left|E_{2}\right|}}}{-\frac{1}{16} \sqrt{\left|E_{1}\right|} \frac{1}{\sqrt{\left|E_{2}\right|}}}=1 .
$$

The simple wave solution then starts at the leading edge characteristic $u=v$. It is thus possible to match with the boundary condition on the leading characteristic and obtain the parameter $-E_{1}$ by applying the relation

$$
E_{0}=\frac{\partial \phi}{\partial u}+\frac{\partial \phi}{\partial v}=4 \sqrt{-E_{1}} \quad \text { on } \quad u=v .
$$

It now remains to verify that at the trailing edge of the expansion fan the function $-1 / S^{(2)}$ approaches the soliton speed

$$
-\frac{\left|E_{1}\right|-\frac{1}{16}}{\left|E_{1}\right|+\frac{1}{16}}
$$

To verify this it is necessary to evaluate $C^{+}$and $C^{-}$explicitly in terms of standard elliptic integrals. This, together with the explicit expressions for the modulation solution, will be discussed in the next section.

\section{Explicit modulation solution and comparison with numerical solution}

To explicitly construct the modulation solution, we start by calculating $C^{+}$and $C^{-}$ in terms of elliptic integrals. To this end it is convenient to introduce the variable $p$ with the transformation $E_{2}=p E_{1}$, so that $0 \leq p \leq 1$. We begin by evaluating $C^{+}$. 
Let us deform the path $b$ shown in Figure 1 onto the segment $E_{2} \leq E \leq 0$. Since the contour $b$ goes onto the second Riemann sheet, the integral is non-zero. Writing the integral in the standard notation of Abramowitz and Stegun [1], we find

$$
C^{+}=\left|E_{1}\right| \frac{K(p)-E(p)}{K(p)}
$$

where $K(p)$ and $E(p)$ are elliptic integrals of the first and second kinds of modulus squared $p$, respectively.

The evaluation of $C^{-}$is different since one of the integrals in (26) has a pole at $E=0$. To evaluate this integral with a pole, we deform the left-hand end of the path $b$ to $E_{1}$ and the right-hand end to infinity. Let us consider the integral in the upper half plane first. The integral in the numerator of the expression (26) for $C^{-}$thus is transformed into an integral in the range $-\infty<E<E_{1}$ and can be expressed in terms of standard elliptic integrals. The contribution from the integral on the second sheet is evaluated in the same way, and we thus obtain

$$
C^{-}=\frac{K(p)-E(p)}{K(p) \sqrt{p}}
$$

Finally, using the expressions (30) and (31) for $C^{+}$and $C^{-}$in the definition (17) for $S^{(2)}$, the implicit solution of the modulation equations is obtained in the form

$$
\frac{u}{v}=-\frac{1}{S^{(2)}(p)}=-\frac{\sqrt{p} K(p)(1-p)\left|E_{1}\right|-E(p)\left(\sqrt{p}\left|E_{1}\right|-\frac{1}{16}\right)}{\sqrt{p} K(p)(1-p)\left|E_{1}\right|-E(p)\left(\sqrt{p}\left|E_{1}\right|+\frac{1}{16}\right)} .
$$

From this expression for the characteristic speed, it is clear that as $p \rightarrow 0, u / v \rightarrow 1$ as required. Also, as $p \rightarrow 1, K(p) \rightarrow \infty$, but $(1-p) K(p) \rightarrow 0$ [1], and therefore

$$
\frac{u}{v} \rightarrow-\frac{\left|E_{1}\right|-\frac{1}{16}}{\left|E_{1}\right|+\frac{1}{16}}
$$

which is the soliton speed, as is required at the trailing characteristic.

Transforming back to the original variables (taking $\Omega=c=1$ which is just an re-scaling of the variables) using (4) and (8), the implicit solution of the modulation equations is therefore

$$
\frac{t-2 x}{t}=-\frac{1}{S^{(2)}(p)}
$$

It now can be seen from (32) that as $x \rightarrow 0, p \rightarrow 0$ and from (33) that as $p \rightarrow 1$, the lead soliton travels with the speed

$$
\frac{1}{1+\frac{1}{16\left|E_{1}\right|}}
$$

which is slower than the characteristic speed at this point.

To complete the modulation theory solution, the explicit expression for $y$ is needed. From the differential equation (21) for $y$, it can be found that in terms of the original variables $x$ and $t$

$$
\begin{aligned}
& y=\operatorname{sc}\left(2 \sqrt{\left|E_{1}\right|}(1+\sqrt{p})\left[t-\left(1+\frac{1}{16\left|E_{1}\right| \sqrt{p}}\right) x\right], m\right), \\
& \phi=\pi+2 \arctan y
\end{aligned}
$$


where

$$
m_{1}=\left(\frac{1-\sqrt{p}}{1+\sqrt{p}}\right)^{2} .
$$

In this solution, the standard conventions of [1] for the elliptic function sc and the modulus $m=1-m_{1}$ and the complementary modulus $m_{1}$ have been used. The solution for the electric field $\phi_{t}$ then is given in the modulation approximation by

$$
\begin{aligned}
E & =\frac{\partial \phi}{\partial t} \\
& =\alpha \operatorname{dn}\left(2 \sqrt{\left|E_{1}\right|}(1+p)\left[t-\left(1+\frac{1}{16\left|E_{1}\right| \sqrt{p}}\right) x\right], m\right)
\end{aligned}
$$

where

$$
\alpha=4 \sqrt{\left|E_{1}\right|}(1+p) .
$$

The function $p(x, t)$ is obtained by inverting (34). It can be seen from (32), (34), and (37) that as $x \rightarrow 0, p \rightarrow 0$ and $m \rightarrow 0$ and thus $\mathrm{dn} \rightarrow 1$ (see [1]). This recovers, as expected, the direct asymptotic result (28), which determines $E_{1}$ in terms of the boundary value $E_{0}$ as

$$
4 \sqrt{\left|E_{1}\right|}=E_{0} .
$$

As $p \rightarrow 1$, the modulation theory solution (38) becomes the leading soliton in the form

$$
\frac{\partial \phi}{\partial t} \sim 8 \sqrt{\left|E_{1}\right|} \operatorname{sech} 4 \sqrt{\left|E_{1}\right|}\left[t-\left(1+\frac{1}{16\left|E_{1}\right|}\right) x\right]
$$

since $\mathrm{dn} \rightarrow$ sech as $m \rightarrow 1$. This behavior of the modulation theory solution for the boundary-value problem is the same as that obtained for the signalling problem for the Korteweg-de Vries equation by Marchant and Smyth [7].

To determine the accuracy of the modulation theory solution for the boundary-value problem for the Sine-Gordon equation, the solution (38) was compared with numerical solutions of the Sine-Gordon equation. The form of the Sine-Gordon equation used for the numerical solutions was

$$
\frac{\partial^{2} \phi}{\partial t^{2}}+\frac{\partial^{2} \phi}{\partial x \partial t}=-\sin \phi
$$

To solve this equation numerically, it is easiest to write it as a first-order system by introducing the new (characteristic) variable

$$
v=\frac{\partial \phi}{\partial t}+\frac{\partial \phi}{\partial x}
$$

so that the equation becomes

$$
\begin{aligned}
& \frac{\partial \phi}{\partial t}+\frac{\partial \phi}{\partial x}=v \\
& \frac{\partial v}{\partial t}=-\sin \phi
\end{aligned}
$$


Equation (44) then is solved using backward differences in $x$ and forward differences in $t$ to become

$$
\phi(x, t+\Delta t)=\left(1-\frac{\Delta t}{\Delta x}\right) \phi(x, t)+\frac{\Delta t}{\Delta x} \phi(x-\Delta x, t)+\Delta t v(x, t)
$$

where $\Delta t$ and $\Delta x$ are the time and space steps, respectively. The second equation (45) was solved using the second-order trapezoidal method in time

$$
v(x, t+\Delta t)=v(x, t)-\frac{1}{2} \Delta t[\sin \phi(x, t+\Delta t)+\sin \phi(x, t)] .
$$

This numerical scheme is stable if $\Delta t / \Delta x<1$.

The nonlinear equation (34) for $p=p(x, t)$ was solved using the secant method combined with an asymptotic expansion near the singular limit $p=1$ to obtain faster convergence in this limit. The elliptic integrals and elliptic functions were evaluated using approximations as given in Abramowitz and Stegun [1].

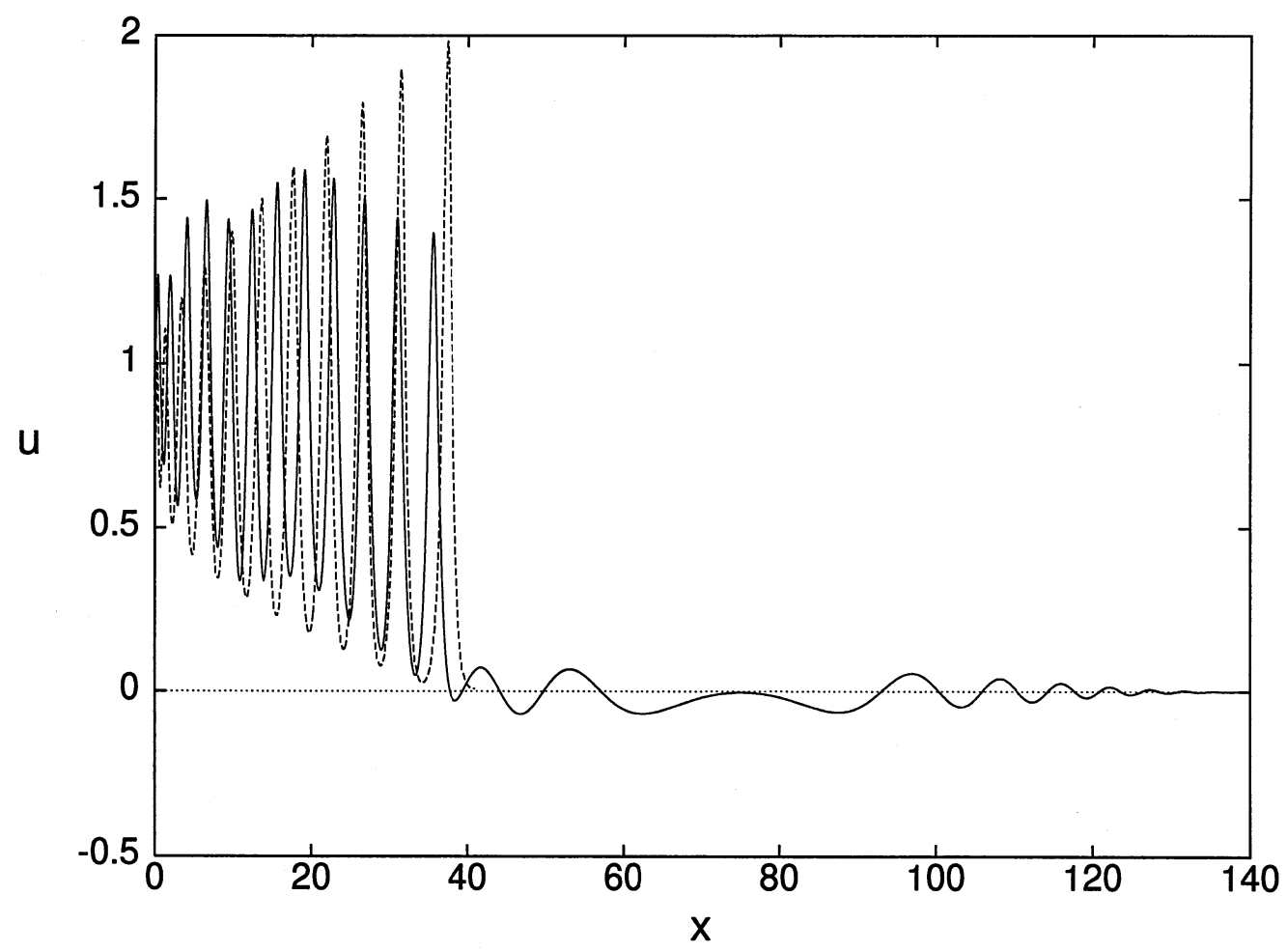

FIGURE 2. Comparison between the numerical solution of the SineGordon equation (42) ( - ) and the modulation theory solution (38) $(---)$. The solutions are shown at $t=75$.

A comparison between the modulation and numerical solutions at $t=75$ is shown in Figure 2, for which $E_{0}=1.0$. The comparison shown in this figure is typical of that for a wide range of boundary values $E_{0}$ and final times $t$. It can be seen that there is good agreement in the envelope of the solution and in the number of 
modulated waves. Unlike the similar modulation solution for the $\mathrm{KdV}$ equation [3, 4], the leading wave of the modulated wavetrain does not match smoothly into the zero state ahead. This is to be expected since the Sine-Gordon equation is hyperbolic and the characteristic speed is larger than the speed of the solitary wave at the leading edge of the modulated wavetrain. The solution in the region between the leading characteristic and the leading soliton is the result of the nonlinear evolution of the waves generated by the discontinuous start-up of the signalling boundary condition. The possibility of describing the waves in this region by another special solution of the integrable PDE (Sine-Gordon equation) has not yet been explored.

In conclusion, we have shown that the solution for a special signalling problem can be approximated by a modulated train of kinks of the Gurevich-Pitaevskii type [4]. This approximate solution extends the exact inverse scattering solution for a signalling problem with a signal of finite duration to one with a signal of infinite duration.

To make the present analysis rigorous, one may try to apply the Lax-Levermore theory [6] to the present problem. That theory allows the possibility of the appearance of new phases. However, numerical solutions show no evidence for the appearance of a new phase when the boundary condition of the signalling problem is held constant.

Acknowledgements. A. A. Minzoni would like to thank the Royal Society of London and the National Academy of Science of Mexico for a travel grant to the University of Edinburgh that made this work possible.

\section{References}

1. M. Abramowitz and I. A. Stegun, Handbook of Mathematical Functions, Dover, New York, 1972.

2. M. G. Forest and D. W. McLaughlin, Modulations of Sinh-Gordon and Sine-Gordon wavetrains, Stud. Appl. Math. 68 (1983), 11-59.

3. B. Fornberg and G. B. Whitham, A numerical and theoretical study of certain nonlinear wave phenomena, Phil. Trans. Roy. Soc. Lond. A 289 (1978), 373-403.

4. A. V. Gurevich and L. P. Pitaevskii, Nonstationary structure of a collisionless shock wave, Sov. Phys. JETP 38 (1974), 291-297.

5. G. L. Lamb, Elements of Soliton Theory, Wiley, New York, 1980.

6. P. D. Lax and D. Levermore, The small dispersion limit of the Korteweg-de Vries equation I, Comm. Pure Appl. Math. XXXVI (1983), 253-290.

7. T. R. Marchant and N. F. Smyth, Initial-boundary value problems for the Korteweg-de Vries equation, IMA J. Appl. Math. 47(3) (1991), 247-264.

8. G. B. Whitham, Linear and Nonlinear Waves, Wiley, New York, 1974.

Department of Mathematics and Mechanics, IIMAS, National Autonomous University of Mexico, Apdo. 20-726, Del. Alvaro Obregon, 01000 D.F. Mexico, Mexico

Department of Mathematics and Statistics, The King's Buildings, University of Edinburgh, Edinburgh, Scotland, EH9 3JZ, U.K. 